\title{
BARRERAS CULTURALES EN LA COMUNICACIÓN Y ÉTICA EN LA GESTIÓN DE RELACIONES PÚBLICAS DE EMPRESAS MULTINACIONALES TURÍSTICAS EN CUBA: UM ESTUDO DE CASO
}

\author{
CULTURAL BARRIERS IN COMMUNICATION AND ETHICS IN THE \\ MANAGEMENT OF PUBLIC RELATIONS OF MULTINATIONAL TOURISM \\ COMPANIES IN CUBA: A CASE STUDY
}

Recebimento: 8/9/2016- Aceite: 15/5/2017- Publicação: 30/06/2017
Processo de Avaliação: Double Blind Review

Iramis Bello Cordero ${ }^{1}$

Licenciada en Educación, Especialista en Comunicación Social

Profesora Auxiliar Facultad de Turismo, Universidad de la Habana, Cuba

\section{Felipe Chibás Ortiz}

Livre Docente pela Escola de Comunicações e Artes da Universidade de São Paulo

Doutorado em Ciências da Comunicação

Universidade de São Paulo

\section{RESUMEN}

Cuba está abriendo paso al desarrollo de la inversión extranjera, la cual, y a partir de las transformaciones económicas, políticas y sociales que se despliegan en el país, tiene una gran importancia. Conocer las características de la gestión en las instalaciones de la nación, bajo la administración mixta, es una tarea vital si se desea alcanzar la eficiencia y eficacia en la práctica de la actividad turística. Ello requiere un abordaje multi e interdisciplinario, en tanto favorece la interrelación de procesos de comunicación intercultural que pueden resultar positivos o nocivos para el devenir de la sociedad y la cultura organizacional cubanas. Este trabajo caracteriza la implementación de las Relaciones Públicas, en los Hoteles Parque Central e Iberostar Varadero, ambos, bajo la gerencia multinacional de la cadena hotelera española Iberostar Hotels and Resorts en asociación con la Cadena nacional Cubanacán, explorando las Barreras Culturales en la Comunicación que pueden entorpecer sus objetivos estratégicos. También se presta especial atención a los aspectos éticos de la gestión de la comunicación de dos hoteles estudiados. Se utilizó el método de estudio de caso de forma principalmente cualitativa. Se aplicaron las técnicas de observación estructurada, encuestas semiestructuradas, revisión bibliográfica, revisión documental y entrevista en profundidad para esclarecer dudas. A través de los resultados levantados se percibió la importancia de conocer las Barreras Culturales en la Comunicación para administrar de forma eficiente los hoteles, principalmente cuando se trata de una red multinacional operando en otro país.

Palabras Claves: Barreras Culturales en a Comunicación, Turismo, Gestión multinacional, Relaciones Públicas, Redes hoteleras, Iberostar.

\footnotetext{
1 Autor para correspondência: Universidad de La Habana: San Lázaro y L. Municipio Plaza de la Revolución. Cuba. 


\section{ABSTRACT}

Cuba is opening the way to the development of foreign investment, which, starting from the economic, political and social transformations that unfold in the country, is of great importance. Knowing the characteristics of the management in the facilities of the nation, under the mixed administration, is a vital task if you want to achieve efficiency and effectiveness in the practice of tourism. This requires a multidisciplinary and interdisciplinary approach, as it favors the interrelation of intercultural communication processes that can be positive or harmful to the future of Cuban society and organizational culture. This work characterizes the implementation of Public Relations in the Hotels Parque Central and Iberostar Varadero, both under the multinational management of the Spanish hotel chain Iberostar Hotels and Resorts in association with the Cubanacan national chain, exploring the Cultural Barriers to Communication that Can hinder their strategic objectives. Special attention is also given to the ethical aspects of the communication management of the two hotels studied. The case study method was used mainly qualitatively. The techniques of structured observation, semistructured surveys, bibliographic review, document review and in-depth interview were used to clarify doubts. Through the results obtained, it was perceived the importance of knowing the Cultural Barriers to Communication to efficiently manage the hotels, mainly when it is a multinational network operating in another country.

Key words: Cultural Barriers to Communication, Tourism, Multinational Management, Public Relations, Hotel Networks, Iberostar.

\section{INTRODUCCIÓN}

La relación de los individuos y su constante comunicación con el fin del entendimiento mutuo es una de las actividades humanas que acompañan el surgimiento de la civilización y la vida en sociedad.

Analizando la comunicación desde su fundamento social, es imprescindible hablar de un proceso dinámico, que se ejecuta desde la micro estructura hasta los segmentos más amplios y complejos de la sociedad, posibilita el comportamiento homogéneo entre los seres humanos que interactúan en las diferentes agrupaciones de la comunidad e influye en los éxitos o fracasos de los grupos y de los individuos que los componen (TRELLES, 2005).

Generalmente, los grupos cuya comunicación es mayor, se expresan con madurez y superior operatividad (AGUILAR, 2007). Las dificultades en la comunicación son fuente permanente de conflictos, pobre desempeño, insatisfacciones de sus integrantes y hasta de la desintegración de las organizaciones (CHIBÀS ORTIZ, 2015). 
Pensar la comunicación como eje del sistema nervioso de una organización, implica la elaboración de estrategias claras que partan de un conocimiento profundo de los individuos que le dan vida, de sus escarmientos y expectativas, sus potencialidades y limitaciones, proyectos de vida, frustraciones, no sólo en su desempeño profesional o laboral, sino en todos los ámbitos de su desarrollo.

De Comunicación Organizacional comienza a hablarse en los años cincuenta del pasado siglo, pero no es hasta la década de los setenta con la publicación del libro “Comunicación en la Organización", del especialista norteamericano Charles Redding cuando, por primera vez, se aborda el término de manera teórica abriendo así un nuevo camino en el campo de las ciencias sociales (GARCÍA, 2012).

La tendencia contemporánea para integrar áreas de la comunicación que funcionaban de manera inconexa como la publicidad, las relaciones públicas, el mix de comunicación del marketing, entre otras, ha propiciado el surgimiento de dicha disciplina, razón por la que resulta de vital referencia en trabajos cuyos temas se enfocan en cualquiera de sus áreas de competencia (KUNSCH, 2006).

La Comunicación Organizacional es una de las herramientas con las que cuenta una empresa para lograr sus objetivos. Se basa en el estudio y gestión de los diferentes flujos de información, en distintos niveles y segmentos de públicos (internos o externos), con el objetivo de favorecer la eficiencia y la eficacia empresariales. Integra métodos y procedimientos de diferentes disciplinas y ciencias sociales, para evaluar y proyectar los procesos que dependen del factor humano (KUSCH, 2006, CHIBÁS ORTIZ, 2015).

Las Relaciones Públicas, como disciplina, tienen un origen anterior a la Comunicación Organizacional, aparecen en el mundo corporativo, como una herramienta de rectificación y conformación de imagen y evolucionan hacia nuevos enfoques y regiones del mundo desarrollado primeramente, hasta su introducción en Latinoamérica durante la década de los 50 del pasado siglo XX (KUSCH, 2006).

El objetivo general que pretendemos es evaluar las Barreras Culturales en la Comunicación presentes en el modelo de Relaciones Públicas empleado por la gerencia multinacional de Iberostar en el sector turístico cubano en correspondencia con el nuevo contexto internacional y de Cuba en particular. Entre los objetivos específicos está el de contribuir a esclarecer las bases teórico-conceptuales relacionadas con las Barreras Culturales en la Comunicación en la gestión de Relaciones Públicas en entidades turísticas a nivel global y en el contexto cubano; así como sus particularidades en Revista ENIAC Pesquisa, Guarulhos (SP),V.6, n.1, jan.- jun. 2017 
gerencias multinacionales. También evaluar aspectos necesarios de introducción y/o modificación en la gestión de dichas entidades, así como identificar las mejores prácticas factibles de generalización.

\section{RELACIONES PÚBLICAS EN CUBA}

En Cuba, las Relaciones Públicas como disciplina y como actividad profesional han atravesado un irregular comportamiento, plagado de incomprensiones conceptuales e ideológicas que, sin obviar la consabida influencia de prácticas mercantilistas y globalizadoras, lastran hoy la buena salud de sus resultados, tanto en el desempeño empresarial, como en la administración pública (MURUAGA, et. al 1998; GARCÍA, 2012).

La pérdida de sus verdaderos objetivos, la trastocaciòn con actividades como divulgación y propaganda, la desatención a los públicos internos, la improvisación e incoherencia en la proyección de una imagen positiva en los públicos externos aún persisten, no obstante los avances teóricos y prácticos que ya se pueden mostrar, iniciados en la década de los 90 (TROCONES, 2011; IGLESIAS, 2013).

Con el desarrollo de empresas de gerencia multinacional comienza un proceso de interculturalidad que involucra a trabajadores nacionales y extranjeros bajo nuevas formas de hacer; se interrelacionan los paradigmas corporativos de las cadenas inversoras en ajuste con las exigencias de la realidad sociocultural y política de Cuba (MURILLO, 2013).

La inversión extranjera y la administración compartida como nuevas modalidades de explotación turística, atraen al país nuevas prácticas en el orden sociocultural que se imbrican en el quehacer de los colectivos laborales, compartiendo hábitos, tradiciones y modus operandis en busca de los resultados económicos planteados que no deben entrar en contradicción con los paradigmas del sistema sociopolítico cubano (MURILLO, 2013; RUZ, 2014).

Para ello, las Relaciones Públicas constituyen una herramienta importante; sin embargo, es necesario profundizar en las regularidades del modelo empleado por los inversionistas extranjeros y/o en los procesos de negociación e interculturalidad que se producen entre ambas gerencias, caso administración compartida, para poder contribuir a la modificación de aquellos rasgos que entorpezcan un desarrollo armonioso y próspero, compatibles con el futuro al que se aspira (GARCÍA, 2012). 
Este trabajo concentra su objeto de estudio en las Barreras Culturales en la Comunicación que caracterizan el modelo de gestión de Relaciones Públicas, específicamente desempeñadas en el sector turístico (CHIBÀS ORTIZ, 2005), atendiendo a la necesidad impostergable de su perfeccionamiento, dados los desafíos inmediatos y perspectivas de crecimiento planteados al país, a partir del incremento del número de turistas extranjeros que paso a ser en el 2016 de más de seis millones.

Es bastante frecuente las investigaciones cuyos precedentes se enfocan en áreas específicas como, la comunicación interna, la comunicación externa, la imagen corporativa en entidades de restauración y alojamiento (RIZO, M.; Romeu 2006; ORTIZ, 2001), entre otras, pero ninguna se ha propuesto la determinación del modelo de gestión de las relaciones públicas asociado a las Barreras Culturales en la Comunicación y destacando dentro de este los aspectos éticos, especialmente en estas nuevas formas de administración mixta de cubanos con extranjeros.

Las Barreras Culturales a la Comunicación estudiadas fueron por Chibás Ortiz (2005) en hoteles cubanos y brasileños:

1. Etnocentrismo. (Valorización extrema del origen del país o cultura versus No valorización)

2. Tendencia al individualismo versus colectivismo

3. Sexismo (masculinidad versus feminidad, es decir un sexo tiene la razón y el otro esta errado)

4. Sensualismo (explícita o implícita exaltación del cuerpo físico) versus no Sensualismo

5. Religiocentrismo (Tendencia a creer que mi religión es la única correcta y las demás están erradas versus Tendencia a pensar que todas las religiones tiene su espacio )

6. Tendencia a sobrevalorar lo urbano o lo rural, según el caso en detrimento del otro polo.

7. Relación destorcida con la ética (Tendencia a tener un respeto rígido por las reglas versus Tendencia a no respetar las reglas)

8. Tendencia a sobrevalorar versus no valorar la edad

9. Adopción de estilos de comunicación autocráticos versus excesivamente laizer faire (dejar hacer todo ) 
10. Tendencia a lo inmediato versus tendencia a lo mediato (Querer los resultados inmediatamente versus Tendencia a querer los resultados a largo plazo)

11. Distancia jerárquica(Valorar mucho los cargos superiores, verse distante de ellos) versus No darle importancia a el cargo que ocupa la persona (verse próximo de los jefes y subordinados).

12. Control de Incerteza (Tendencia a querer controlar la incertezas, planificar las vida versus a dejar que las cosas sucedan sin planificarlas)

13. Tendencia a la internalidad versus externalidad (Tendencia a atribuir las causas del comportamiento a si mismo versus Tendencia a atribuir la causa del éxito o los problemas a los demás, a lo externo).

\section{2. ÉTICA Y BARRERAS CULTURALES EN LA COMUNICACIÓN}

Es ahora bastante frecuente también la investigación de los aspectos éticos vinculados a la gestión (DEBELJUH.2009) y cada vez más también relativos a la comunicación organizacional (ARREDONDO TRAPERO, Florina; GARZA GARCÍA, Jorge; VILLA CASTAÑA, 2014). Pero pocas investigaciones y textos relacionan los aspectos éticos a los aspectos culturales que envuelven personas que trabajen en multinacionales o empresas que reúnan culturas diversas (SANTOS, 2014).

Podemos entender la ética organizacional en los marcos de este artículo, como el conjunto de principios y disposiciones enfocadas para la acción, históricamente producidos, cuyo objetivo es fundamentar las acciones humanas en las empresas (PATRUS-PENA; CASTRO, 2010). Es justamente en la convivencia organizacional y comunitaria, sea esta presencial o virtual, que el ser humano se descubre y se realiza en cuanto un ser moral. Es en la relación con el otro que surgen los problemas, Barreras Culturales en la Comunicación, conflictos y las indagaciones morales en lo cotidiano o en la empresa:

La principal cuestión de investigación que tratamos de respondernos es entender cuáles son las principales Barreras Culturales en la Comunicación presentes en el modelo de gestión de Relaciones Públicas empleado en la gerencia multinacional del sector turístico-hotelero de la red multinacional Iberostar en Cuba. Dentro de ese todo general los aspectos éticos de la gestión asociada a las Barreras Culturales de la Comunicación tendrán también especial atención. Pretendemos de esa manera, de Revista ENIAC Pesquisa, Guarulhos (SP),V.6, n.1, jan.- jun. 2017 
contribuir al perfeccionamiento de la gestión hotelera, así como a la generalización de las buenas prácticas del sector.

Pensamos que la evaluación de las Barreras Culturales en el modelo de gestión de Relaciones Públicas empleado en el sector turístico cubano con inversión extranjera permitirá, tener una mayor precisión en la introducción y/o modificación, así como en la identificación de buenas prácticas de servicio, válidas para su generalización y pertinentes, así como en correspondencia con el nuevo contexto de incremento considerable del turismo extranjero en Cuba.

\section{GRUPO IBEROSTAR}

Cuba se encuentra hoy en un proceso de cambio, abriéndose al turismo y a la inversión extranjera, recibiendo en el año de 2016, más de cuatro millones de turistas extranjeros (INFOTUR, 2017).

El Grupo Iberostar, es un conjunto de empresas dedicadas al sector turístico con sede en Palma de Mallorca, España. Actualmente, este grupo está formado por, Iberostar Hotels \& Resorts (división hotelera), Iberoservice Incoming Services (división receptiva) e Iberostate (división inmobiliaria). Junto con el Grupo Barceló, Sol Meliá y RIU Hotels forma parte de las cuatro grandes cadenas hoteleras baleares del mundo.

La historia del Grupo Iberostar se remonta a más de 120 años y tiene un germen común con las empresas de calzado Camper y Lotusse. En 1877 Antonio Fluxá, artesano zapatero, zarpó rumbo a Inglaterra para estudiar la producción industrial del calzado. A su regreso en Mallorca, reunió a un grupo de artesanos de la comarca de Inca y fundó la primera industria de calzado de Baleares. Este espíritu innovador y emprendedor se ha ido transmitiendo a generaciones futuras, con principios tales como: amor a la profesión, honradez, austeridad y oficio de calidad. A partir de ese momento, fue la familia Fluxá la primera en emplear una marca comercial para sus zapatos: Lotusse.

Lorenzo Fluxá Figuerola se hizo cargo a los 21 años de la fábrica de su padre, y continuó con la expansión internacional. En 1956 compró una pequeña agencia de viajes con 6 oficinas y 40 empleados, llamada Viajes Iberia, e inició su desarrollo. Este inculcó a sus hijos desde su infancia la siguiente máxima: “Tenéis que ser internacionales".

Tiene más de 14 millones de clientes. Sus más de 23.000 empleados en todo el mundo trabajan cada día para mantener vivas estas señas de identidad, de los cuales, 
trabajan en la división hotelera, el 80\%, que representa una cantidad de 16.300 empleados. Factura al año más de 300 MII (Mil Millones) $€$, y el accionariado se divide en el $60 \%$ perteneciente a la familia Fluxá, y el $40 \%$ restante perteneciente al grupo TTOO (Turoperador) Thomas Cook.

\section{IBEROSTAR EN CUBA}

Según la señora González Gutiérrez, directora comercial de Iberostar Hoteles en Cuba, esta nación caribeña es uno de esos destinos donde Iberostar ve mayores perspectivas y posibilidades de desarrollo turístico (BÁEZ 2014). En este, dicho grupo administra 10 hoteles actualmente, entre los que se encuentran:

- Iberostar "Grand Hotel Trinidad", en la ciudad de Trinidad, con 40 habitaciones; Iberostar "Parque Central", en la Habana Vieja, con 427 habitaciones;

- Iberostar "Varadero", en Matanzas, Varadero, con 386 habitaciones;

- Iberostar "Laguna Azul”, también en Varadero, con 814 habitaciones;

- Iberostar "Taínos", también en Varadero, con 272 habitaciones;

- Iberostar "Playa Alameda", también en Varadero, con 391 apartamentos;

- Iberostar "Daiquirí”, en Cayo Guillermo, con 312 habitaciones;

- Iberostar "Ensenachos", en Cayo Santa María, con 460 suites en bungalows y 46 villas con jacuzzi;

- Iberostar "Cayo Coco", en Cayo Coco, con 338 habitaciones;

- Iberostar "Mojito", también en Cayo Coco, con 352 habitaciones; para un total en Cuba, de 3965 habitaciones. Iberostar ve a Cuba como un destino seguro, con gran diversidad en sí mismo, y muchas perspectivas en cuanto a desarrollo turístico se refiere. Según la señora González:

Muchos de los grandes grupos españoles y mundiales de la industria hotelera también se encuentran aquí, lo que confirma la importancia de esta plaza turística". Los principales mercados con los que trabaja para este país son, en primer lugar Canadá, seguido de Alemania, los Países Bajos, Inglaterra y luego España, Italia y Francia(BÁEZ 2014),.

Esta cadena hotelera tiene como perspectivas en Cuba, para los próximos 5 años: 
- - Tener de 16 a 18 Hoteles Iberostar en Cuba.

- - Que todos los Hoteles Iberostar en Cuba sean 5 estrellas.

- - Aumentar la cantidad de hoteles en la Habana, los Cayos (playa Pilar) y Holguín (Guardalavaca).

- - Para el 2016 tener un hotel más, tanto en la Habana, como en Varadero.

- - Para el 2017, otro en Playa Pesquero y Ancón.

\section{PROCEDIMIENTOS METODOLÓGICOS}

Siguiendo los criterios de Sampieri (2006) La investigación se realizó en el periodo de los años 2014 a 2015 con un enfoque predominantemente cualitativo, por su alcance exploratoria y descriptiva, pues se realiza un corte transversal del estado actual de los procesos de comunicación en entidades turísticas donde se implementa la inversión extranjera o la administración compartida, sin pretender influir en los fenómenos que se vayan descubriendo ni intervenir la práctica organizacional, con un diseño No Experimental transversal descriptivo.

Se empleó una combinación de métodos y procedimientos cualitativos y cuantitativos para procesar y analizar los resultados de las técnicas aplicadas en aras de un enfoque más integral. No se aspira obtener novedades teóricas, sino aportar resultados dirigidos a la toma de decisiones.

Se empleó especialmente el sistema de categorías y la metodología aportada por Chibás Ortiz $(2005 ; 2015)$ en su texto Creatividad, Comunicación y Cultura. Gestión innovadora de proyectos educativo-culturales en la era digital, que propone una categorización de 13 Barreras Culturales en la Comunicación, probada en varios países.

Se utilizó la metodología de estudio de caso. Se aplicaron las técnicas de observación estructurada, encuestas semiestructuradas, revisión bibliográfica, revisión documental y entrevista en profundidad para esclarecer dudas.

\subsection{VARIABLE DEPENDIENTE PLANTEADA}

La variable dependiente de este trabajo es el modelo de gestión de las Relaciones Públicas en los Hoteles Iberostar Parque Central y Varadero, teniendo además como principal foco de la pesquisa, las Barreras Culturales en la Comunicación que se manifiestan en él, asociadas a la ética, entre otras variables. 


\subsection{VARIABLES INDEPENDIENTES ANALIZADAS}

Siguen las variables independientes analizadas y sus indicadores en el Cuadro

$\mathrm{N}^{\circ} .1$.

\begin{tabular}{|c|c|}
\hline Variables independientes & Indicadores \\
\hline $\begin{array}{l}\text { 1. Generalidades } \\
\text { sobre la gestión de las } \\
\text { relaciones públicas }\end{array}$ & $\begin{array}{l}\text { Condiciones materiales, presupuesto, archivo del profesional y } \\
\text { subordinación }\end{array}$ \\
\hline $\begin{array}{l}\quad \text { Barreras } \\
\text { Culturales en la } \\
\text { Comunicación }\end{array}$ & $\begin{array}{l}\text { Se estudiaron las } 13 \text { Barreras Culturales en la Comunicación } \\
\text { propuestas por Chibás Ortiz (2005). A saber: Etnocentrismo, } \\
\text { Tendencia al individualismo versus colectivismo, Sexismo } \\
\text { Sensualismo, Religiocentrismo, Tendencia a sobrevalorar lo urbano } \\
\text { o lo rural, Relación destorcida con la ética, Tendencia a sobrevalorar } \\
\text { versus no valorar la edad, Adopción de estilos de comunicación } \\
\text { autocráticos versus excesivamente laizer faire, } \\
\text { Tendencia a lo inmediato versus tendencia a lo mediato, } \\
\text { Distancia jerárquica, Control de Incerteza y } \\
\text { Tendencia a la internalidad versus externalidad }\end{array}$ \\
\hline $\begin{array}{l}\text { 3. Modelos de las relaciones } \\
\text { públicas }\end{array}$ & $\begin{array}{l}\text { Objetivos, naturaleza de la comunicación y retroalimentación } \\
\text { percibidos en la gestión de la disciplina }\end{array}$ \\
\hline 4. Ética & $\begin{array}{l}\text { Valores organizacionales, normas y reglas según estos, formas de } \\
\text { enfrentar situaciones complejas, incentivos a los trabajadores } \\
\text { destacados y desarrollo de las potencialidades de todos los } \\
\text { trabajadores }\end{array}$ \\
\hline 5. Funciones & $\begin{array}{l}\text { Aseguramiento de una comunicación bidireccional con los públicos, } \\
\text { relación con las áreas de marketing y promoción, información y } \\
\text { asesoramiento a la dirección, trabajo con el público interno, } \\
\text { equilibrio entre la responsabilidad social y los intereses de la } \\
\text { instalación, participación en la toma de } \\
\text { decisiones estratégicas, recepción y trámites de quejas, control de la } \\
\text { identidad e imagen corporativas, trabajo con la opinión pública y } \\
\text { líderes de opinión, relación con los medios y órganos de } \\
\text { comunicación masiva, participación en la introducción de nuevos } \\
\text { productos, servicios y proyectos, relaciones con las organizaciones } \\
\text { políticas y de masas, y planeación y coordinación de actividades y } \\
\text { eventos }\end{array}$ \\
\hline 6. Perfil del relacionista público & $\begin{array}{l}\text { Conocimientos profesionales de la actividad de comunicación; } \\
\text { procedencia académica; interés por la superación; período de } \\
\text { experiencia en el medio y nivel de conocimientos sobre su } \\
\text { organización; conocimientos idiomáticos; habilidades en } \\
\text { comunicación (nivel cultural, dicción y expresión oral); presencia } \\
\text { personal; y utilización de medios informáticos }\end{array}$ \\
\hline 7. Segmentación de los públicos & $\begin{array}{l}\text { Definición del público interno; definición de los públicos externos; y } \\
\text { decantación de públicos especiales }\end{array}$ \\
\hline 8. Investigación & $\begin{array}{l}\text { Frecuencia; diagnósticos de comunicación y estudios de opinión } \\
\text { pública; y evaluación de la imagen de la compañía con auditorías de } \\
\text { imagen }\end{array}$ \\
\hline 9. Acciones & $\begin{array}{l}\text { Utilización de textos; organización de eventos, internos y externos; } \\
\text { patrocinio de actividades en la comunidad; uso de la fotografía; } \\
\text { protocolo y ceremonial; uso de soportes informáticos; y trabajo con } \\
\text { los líderes de opinión }\end{array}$ \\
\hline 10. Comunicación & $\begin{array}{l}\text { Política de comunicación interna y externa; canales más usados; } \\
\text { bidireccionalidad de la comunicación; y principales portavoces de la } \\
\text { instalación }\end{array}$ \\
\hline
\end{tabular}

Cuadro No. 1 Variables independientes de la investigación

Revista ENIAC Pesquisa, Guarulhos (SP),V.6, n.1, jan.- jun. 2017 
Fuente: Archivo de los autores

\subsection{HOTELES ESTUDIADOS}

Dados los objetivos planteados para el presente estudio, que eran referentes a entender el modelo de gestión de las relaciones públicas, la ética y las Barreras Culturales en la Comunicación de una red hotelera multinacional que operase en /cuba, se seleccionó por el sistema de muestra por conveniencia, dos hoteles de la red turística Iberoestar. Los hoteles seleccionados fueron el Iberoestar Parque Central y el Iberoestar Varadero, por ambos de los que lideran en número de huéspedes, tasa de ocupación y de satisfacción de los huéspedes, así como por ser de fácil acceso. Ellos según los datos levantados y las entrevistas realizadas, representarían adecuadamente la forma de gestión de esa red. A seguir comentamos las características principales de los mismos.

\section{Hotel Iberostar Parque Central}

El moderno y lujoso hotel Iberostar Parque Central se encuentra ubicado en el corazón de la Habana Vieja, justo al frente del Parque Central del cual toma su nombre. Fue construido en 1998 con una última reforma en 2010.Tiene la categoría de Hotel de Ciudad. Se dirige principalmente a los segmentos de mercado de familia, negocios y cultural.

Muestra una propiedad moderna que combina el lujo del estilo colonial con servicios de infraestructura flamante, una auténtica mezcla del pasado con el futuro dispuesta con elegancia y estilo, sin lugar a dudas, una de las mejores opciones de alojamiento en la Habana. Posee un total de 380 trabajadores.

\section{Hotel Iberostar Varadero}

El Hotel Iberostar Varadero se encuentra situado en: Punta Francés, Varadero. Carretera Las Morlas Km. 16, Sector Punta Hicacos, Varadero, Cuba. Tiene la categoría de Hotel de Playa.

El hotel se inauguró en 2004, consta de 11 edificios con un total de 386 habitaciones, de las cuales 4 están adaptadas para personas discapacitadas, 324 habitaciones son de categoría estándar, 60 son suites júnior y 2 suites presidenciales.

Iberostar Varadero se ha especializado en un segmento de mercado conformado principalmente por grupos familiares, compuestos por individuos de diversas edades, entre las que sobresalen las parejas jóvenes con niños, con una buena solvencia económica que proceden principalmente de Europa (Inglaterra, Alemania) y Canadá. 
Estas personas buscan los atractivos de sol y playa y manifiestan una tendencia a interesarse por el turismo ecológico.

\subsection{POBLACIÓN Y MUESTRA UTILIZADAS PARA LA PESQUISA.}

Fue entendida como la población total los trabajadores cubanos y extranjeros que participan de la gerencia mixta en Cuba entre Iberostar, Gran Caribe y Cubanacán. De ellos se seleccionó como muestra por conveniencia 42 trabajadores, tanto nacionales como extranjeros, de los Hoteles Iberostar Parque Central y Varadero. Este muestreo fue intencional puro, ya que la muestra fue escogida de manera intencional en función de los criterios de selección considerados por los investigadores a saber: más de 3 años de permanencia en la entidad hotelera.

\subsection{CARACTERÍSTICAS DE LA MUESTRA}

Los Hoteles Iberostar Parque Central y Varadero se distinguen por la excelencia de la gestión y sus aportes económicos al país, portando reconocimientos del Ministerio de Turismo de Cuba y del Sindicato de Trabajadores del ramo. La muestra, estuvo compuesta por Directivos y trabajadores en general, tanto de los Hoteles como de la cadena, atendiendo a la existencia de representatividad de todas las áreas claves y de apoyo de estas entidades, así como una permanencia de más de 3 años en el puesto de trabajo.

\subsection{RESULTADOS OBTENIDOS DEL TRABAJO DE CAMPO.}

A seguir mostramos el Cuadro No.2 con una síntesis de la gestión de las Relaciones Públicas en los hoteles Iberostar Parque Central e Iberostar Varadero administrados por esa red en asociación con la cadena Cubanacán, de acuerdo con las variables independientes enfocadas, a través de los instrumentos de investigación utilizados.

\begin{tabular}{|c|c|c|}
\hline $\begin{array}{l}\text { Variables } \\
\text { Independientes }\end{array}$ & Varadero & a $\mathrm{C}$ \\
\hline $\begin{array}{l}\text { 1. } \\
\text { Generalidades } \\
\text { sobre la gestión } \\
\text { de las } \\
\text { Relaciones }\end{array}$ & $\begin{array}{l}\text { En el Hotel se conoce en un } 100 \% \text { qué son las } \\
\text { Relaciones Públicas, los objetivos de la } \\
\text { disciplina y se considera que los trabajadores } \\
\text { contribuyen, también en un } 100 \% \text {, al } \\
\text { desarrollo de la misma. Esta influye }\end{array}$ & $\begin{array}{l}\text { Las Relaciones Públicas y sus objetivos } \\
\text { son medianamente conocidos en la entidad } \\
(67 \% \text { y } 58 \% \text { respectivamente). Esta } \\
\text { influye un } 75 \% \text { en los servicios del Hotel. } \\
\text { Los trabajadores contribuyen al desarrollo }\end{array}$ \\
\hline
\end{tabular}




\begin{tabular}{|c|c|c|}
\hline Públicas & $\begin{array}{l}\text { totalmente en la calidad de los servicios del } \\
\text { Hotel. } \\
\text { El relacionista público tiene buenas } \\
\text { condiciones de trabajo, no genera gastos } \\
\text { propios por lo que no tiene un presupuesto } \\
\text { asignado, y se subordina al Área de } \\
\text { Alojamiento, particularmente a Recepción, por } \\
\text { lo que no tiene ningún poder de decisión. }\end{array}$ & $\begin{array}{l}\text { exitoso de la actividad. } \\
\text { El relacionista público tiene buenas } \\
\text { condiciones de trabajo, no genera gastos } \\
\text { propios por lo que no tiene un presupuesto } \\
\text { asignado, y se subordina al departamento } \\
\text { de calidad, tiene poder de decisión sobre } \\
\text { los clientes. } \\
\text { Los aspectos que mayormente inciden en } \\
\text { las relaciones interpersonales son el } \\
\text { lenguaje de gestos, el nivel de información } \\
\text { y el conocimiento de la actividad. }\end{array}$ \\
\hline $\begin{array}{l}2 . \quad \text { Barreras } \\
\text { Culturales en } \\
\text { la } \\
\text { Comunicación }\end{array}$ & 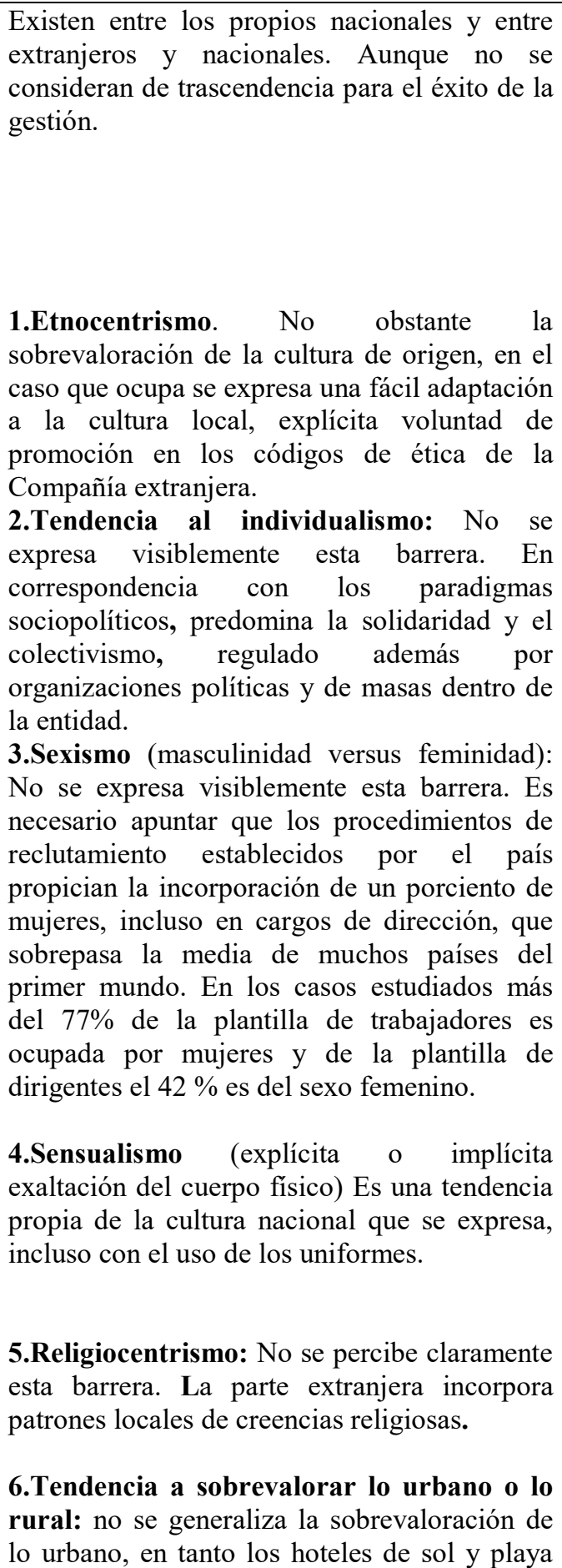 & 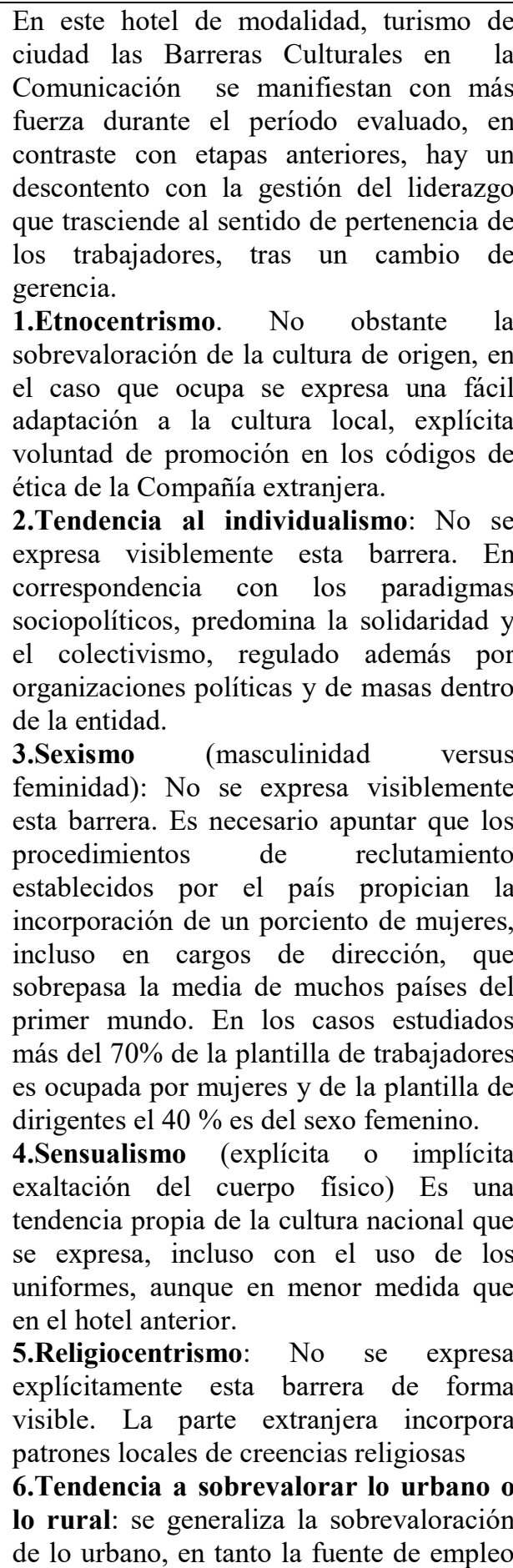 \\
\hline
\end{tabular}




\begin{tabular}{|c|c|c|}
\hline & $\begin{array}{l}\text { constituyen una fuente de empleo segura, } \\
\text { estable que garantizan un nivel de vida } \\
\text { comunitario, más bien se reniega de la } \\
\text { dinámica urbana. } \\
\text { 7.Relación destorcida con la ética: No se } \\
\text { expresa esta barrera. Las normas establecidas } \\
\text { por el Estado cubano, en muchos casos limitan } \\
\text { el desarrollo y la agilidad de la gestión, sin } \\
\text { embargo existe la certeza y la voluntad de } \\
\text { cambio en el colectivo laboral. } \\
\text { 8.Tendencia a sobrevalorar la edad: No se } \\
\text { expresa esta barrera. Existe un respeto por el } \\
\text { trabajador de mayor edad y permanencia, pero } \\
\text { se da paso a los jóvenes talentos. Todos gozan } \\
\text { de los mismos derechos de capacitación y } \\
\text { promoción. } \\
\text { 9.Adopción de estilos de comunicación } \\
\text { autocráticos: No es un indicador } \\
\text { representativo, dado que se generaliza el estilo } \\
\text { colectivo de toma de decisiones, la } \\
\text { sistematicidad y la persistencia, sin embargo, } \\
\text { la parte extranjera siente que se limita su } \\
\text { política de sanciones por las regulaciones } \\
\text { establecidas al efecto por la política de } \\
\text { protección y seguridad al trabajador de la parte } \\
\text { cubana. Aún así hay algunas manifestaciones } \\
\text { de la misma. } \\
\text { 10..Tendencia a lo inmediato versus } \\
\text { tendencia a lo mediato: Este es un indicador } \\
\text { que se dispara ampliamente, es evidente que la } \\
\text { práctica local entra en contradicciones por } \\
\text { problemas por razones ajenas. Predomina la } \\
\text { externalidad. } \\
\text { burocratismo y lentitud en las decisiones. } \\
\text { eficaz, obstaculizada por el cuerpo de } \\
\text { regulaciones locales que aún requieren de } \\
\text { transformaciones necesarias para agilizar el } \\
\text { desarrollo. } \\
\text { 13.Distancia jerárquica: El estilo de } \\
\text { dirección es a puertas abiertas, con un enfoque } \\
\text { de jovialidad y camaradería, pero se crea } \\
\text { distancia jerárquica en tanto las condiciones } \\
\text { financieras y materiales de la parte extranjera } \\
\text { son muy superiores. }\end{array}$ & $\begin{array}{l}\text { 10.Tendencia a lo inmediato versus } \\
\text { tendencia a lo mediato: Este es un } \\
\text { indicador que se dispara ampliamente } \\
\text { igual que en el anterior hotel, es evidente } \\
\text { que la práctica local entra en } \\
\text { contradicciones por burocratismo y } \\
\text { lentitud en las decisiones. } \\
\text { 11.Distancia jerárquica: Aunque el estilo } \\
\text { de dirección es a puertas abiertas, pero se } \\
\text { crea la distancia jerárquica no apenas por } \\
\text { las condiciones financieras y materiales de } \\
\text { la parte extranjera son muy superiores, } \\
\text { sino también porque los nuevos gestores } \\
\text { no son muy aceptados por los funcionarios } \\
\text { o trabajadores. } \\
\text { 12.Control de Incerteza La planeación } \\
\text { existe y se exige pero no es lo } \\
\text { suficientemente eficaz, obstaculizada por } \\
\text { el cuerpo de regulaciones locales que aún } \\
\text { requieren de transformaciones necesarias } \\
\text { para agilizar el desarrollo. } \\
\text { 13.Tendencia a la internalidad versus } \\
\text { externalidad: Se tiende a justificar los } \\
\text { problemas por razones ajenas. Predomina } \\
\text { la externalidad. }\end{array}$ \\
\hline $\begin{array}{l}\text { 3. Ética y } \\
\text { planificación }\end{array}$ & $\begin{array}{l}\text { Existe un código de ética en la organización y } \\
\text { los valores que se intentan difundir son } \\
\text { mayormente el compromiso, la eficacia, la } \\
\text { eficiencia, el respeto entre los trabajadores y la } \\
\text { competencia. Las conductas y acciones a }\end{array}$ & $\begin{array}{l}\text { Existe un código de ética en la } \\
\text { organización y los valores que se intentan } \\
\text { difundir son apreciar a los recursos } \\
\text { humanos, el respeto al empleado, el } \\
\text { compromiso con la organización, la }\end{array}$ \\
\hline
\end{tabular}




\begin{tabular}{|c|c|c|}
\hline & $\begin{array}{l}\text { emprender en caso de situaciones de crisis } \\
\text { están planificadas, los trabajadores tienen } \\
\text { conocimiento de esto y participan en ellas, } \\
\text { cada lunes, donde se reúnen con los directores, } \\
\text { con el objetivo de redefinir o solo repasar } \\
\text { dichas estrategias. } \\
\text { Se realizan cumpleaños colectivos y se insiste } \\
\text { en la superación de los empleados. Las } \\
\text { relaciones interpersonales son buenas e } \\
\text { inciden en ellas el conocimiento de la } \\
\text { actividad, el sentido de la autoridad, el buen } \\
\text { uso del tiempo, el lenguaje de los gestos y el } \\
\text { nivel de información. Mantener un buen clima } \\
\text { organizacional es un aspecto muy valorado. } \\
\text { Valores como solidaridad, respeto, confianza, } \\
\text { fidelidad y cooperación están muy presentes. }\end{array}$ & $\begin{array}{l}\text { competencia profesional y la eficacia. Las } \\
\text { conductas y acciones a emprender en caso } \\
\text { de situaciones de crisis están planificadas, } \\
\text { los trabajadores tienen conocimiento de } \\
\text { esto y participan en ellas. Se insiste en la } \\
\text { superación de los empleados. Las } \\
\text { relaciones interpersonales son buenas e } \\
\text { inciden en ellas el nivel de información, el } \\
\text { lenguaje de los gestos, el conocimiento de } \\
\text { la actividad, el buen uso del tiempo y el } \\
\text { sentido de la autoestima } \\
\text { Valores como respeto y confianza son muy } \\
\text { presentes.. }\end{array}$ \\
\hline 4. Funciones & $\begin{array}{l}\text { La función principal del relacionista público } \\
\text { es mediar entre la organización es mediar } \\
\text { entre la organización y sus clientes, } \\
\text { proveedores y comunidad, aunque también es } \\
\text { en menor cuantía, una característica de la } \\
\text { planificación estratégica, un instrumento para } \\
\text { hacer frente a las crisis y una herramienta de } \\
\text { marketing. } \\
\text { Además de encargarse de la recepción y } \\
\text { trámite de las quejas que se presentan sobre el } \\
\text { hotel; el aseguramiento de una comunicación } \\
\text { bidireccional con los públicos; el equilibrio } \\
\text { entre la responsabilidad social y los intereses } \\
\text { de la instalación; el trabajo con la opinión } \\
\text { pública y los líderes de opinión; y las } \\
\text { relaciones con las organizaciones políticas y } \\
\text { de masas. }\end{array}$ & $\begin{array}{l}\text { Las Relaciones Públicas en la entidad son } \\
\text { principalmente un mediador entre la } \\
\text { organización y sus clientes, proveedores y } \\
\text { comunidad; además de una herramienta de } \\
\text { marketing y una característica de la } \\
\text { planificación estratégica. } \\
\text { El relacionista público se encarga también } \\
\text { del aseguramiento de una comunicación } \\
\text { bidireccional con los públicos; el } \\
\text { equilibrio entre la responsabilidad social y } \\
\text { los intereses de la instalación; la } \\
\text { participación en la toma de decisiones } \\
\text { estratégicas de la instalación; la recepción } \\
\text { y trámites de las quejas que se presentan } \\
\text { sobre el hotel; el control de la identidad e } \\
\text { imagen corporativas; trabajo con la } \\
\text { opinión pública y líderes de opinión; } \\
\text { participación en la introducción de nuevos } \\
\text { productos, servicios o proyectos; y la } \\
\text { relación con las organizaciones políticas y } \\
\text { de masas. }\end{array}$ \\
\hline $\begin{array}{l}\text { 5. Perfil del } \\
\text { relacionista } \\
\text { Público }\end{array}$ & $\begin{array}{l}\text { Para poder ocupar este cargo tiene que tener } \\
\text { como mínimo Nivel Superior, debe llevar } 2 \\
\text { años ejerciendo la actividad; conocer } 2 \\
\text { idiomas y ser carismático. }\end{array}$ & $\begin{array}{l}\text { Para poder ocupar este cargo hay que ser } \\
\text { universitario, se debe tener experiencia en } \\
\text { la actividad y conocer } 2 \text { idiomas. No se } \\
\text { exige el carisma. }\end{array}$ \\
\hline $\begin{array}{l}6 . \\
\text { Segmentación } \\
\text { de los públicos }\end{array}$ & $\begin{array}{l}\text { Los públicos están bien definidos entre } \\
\text { proveedores; autoridades del sector, políticas y } \\
\text { gubernamentales; públicos internos } \\
\text { (trabajador), directivos (principal portavoz) y } \\
\text { cliente externo (huésped), siendo este último } \\
\text { el más importante para la gestión de las } \\
\text { Relaciones Públicas en las entidades. También } \\
\text { está la prensa aunque no trabajan con ella } \\
\text { directamente. }\end{array}$ & $\begin{array}{l}\text { Los públicos están bien definidos entre } \\
\text { proveedores; autoridades del sector, } \\
\text { políticas y gubernamentales; públicos } \\
\text { internos (trabajador), directivos (principal } \\
\text { portavoz) y cliente externo (huésped), } \\
\text { siendo este último el más importante para } \\
\text { la gestión de las Relaciones Públicas en las } \\
\text { entidades. También está la prensa aunque } \\
\text { no trabajan con ella directamente }\end{array}$ \\
\hline 7. Investigación & $\begin{array}{l}\text { Se realizan estudios de opinión pública y } \\
\text { auditorías de imagen, cada } 3 \text { meses, por la } \\
\text { compañía mexicana Ges Hotels mediante } \\
\text { encuestas, Internet, etc. Tienen una relativa } \\
\text { autonomía. }\end{array}$ & $\begin{array}{l}\text { Las auditorías de imagen son realizadas } \\
\text { por el Departamento de Calidad. Los } \\
\text { estudios de opinión pública son llevados a } \\
\text { cabo por la compañía mexicana Ges Hotels } \\
\text { mediante encuestas. Tienen menos } \\
\text { autonomía en este punto.. }\end{array}$ \\
\hline 8. Acciones & $\begin{array}{l}\text { El Hotel se vincula con la comunidad } \\
\text { mayormente mediante la donación de bienes, } \\
\text { así como movilizaciones sociales y políticas, }\end{array}$ & $\begin{array}{l}\text { La organización se vincula con la } \\
\text { comunidad mayormente mediante } \\
\text { las movilizaciones sociales y políticas, }\end{array}$ \\
\hline
\end{tabular}

Revista ENIAC Pesquisa, Guarulhos (SP),V.6, n.1, jan.- jun. 2017 


\begin{tabular}{|c|c|c|}
\hline & $\begin{array}{l}\text { actividades infantiles y servicios de premios } \\
\text { gratuitos. } \\
\text { Sus claves del éxito se le atribuyen } \\
\text { principalmente al estilo de dirección, a las } \\
\text { facilidades materiales, a la política de } \\
\text { estímulo, a la formación profesional y a las } \\
\text { relaciones de solidaridad y cooperación. }\end{array}$ & $\begin{array}{l}\text { actividades infantiles y en } \\
\text { menor medida, donaciones de bienes y } \\
\text { para solucionar } \\
\text { contingencias. Sus claves del éxito se le } \\
\text { atribuyen principalmente a } \\
\text { la formación profesional de los } \\
\text { trabajadores y dirigentes, las facilidades } \\
\text { materiales, al estilo de dirección, a la } \\
\text { correspondencia } \\
\text { de prácticas culturales entre ambas } \\
\text { gerencias y a las normas y leyes nacionales }\end{array}$ \\
\hline $\begin{array}{l}9 . \\
\text { Comunicación }\end{array}$ & $\begin{array}{l}\text { La cadena gestiona las relaciones públicas y la } \\
\text { comunicación organizacional en Cuba de la } \\
\text { misma manera que en otros países. La del } \\
\text { hotel se realiza con el objetivo de difundir } \\
\text { información mayormente, así como para } \\
\text { escuchar quejas y necesidades, a través del uso } \\
\text { de medios electrónicos (correo, sitio web, } \\
\text { etc.), vía telefónica, de forma directa y de } \\
\text { todas a la vez. El relacionista público } \\
\text { mantiene una comunicación constante con los } \\
\text { trabajadores así mismo como estos a veces } \\
\text { reciben sus orientaciones. }\end{array}$ & $\begin{array}{l}\text { La comunicación en el hotel se realiza, en } \\
\text { orden descendente de importancia, de } \\
\text { forma directa, a través de un intermediario, } \\
\text { a través de medios electrónicos, de todas } \\
\text { estas formas a la vez y por última, la vía } \\
\text { telefónica. Se comunica con sus públicos } \\
\text { para difundir información, escuchar las } \\
\text { quejas y necesidades de los clientes y para } \\
\text { persuadirlos. El relacionista público } \\
\text { mantiene una comunicación constante con } \\
\text { los trabajadores así mismo como estos a } \\
\text { veces reciben sus orientaciones }\end{array}$ \\
\hline $\begin{array}{l}\text { 10. Modelo de } \\
\text { Relaciones } \\
\text { Públicas }\end{array}$ & $\begin{array}{l}\text { El objetivo del relacionista, es la atención } \\
\text { directa al cliente externo, al que le recepciona } \\
\text { las quejas y sugerencias, las inquietudes, } \\
\text { realiza reservas para los diferentes } \\
\text { restaurantes, así como los informa de los } \\
\text { diferentes servicios y atractivos que brinda el } \\
\text { hotel. Tiene un enfoque de trabajo continuo y } \\
\text { está explícito en planes y programas. La } \\
\text { naturaleza de la comunicación es bidireccional } \\
\text { y se evidencia la retroalimentación mediante } \\
\text { encuestas de satisfacción y llamadas de } \\
\text { cortesía al huésped del hotel, técnicas que } \\
\text { igualmente se utilizan para realizar los } \\
\text { estudios de opinión pública. } \\
\text { Existen relaciones con la comunidad a partir } \\
\text { del cuidado del medio ambiente, el empleo, la } \\
\text { donación de bienes y atención a escuelas. La } \\
\text { disciplina no es considerada mayoritariamente } \\
\text { como una herramienta de marketing y la } \\
\text { organización pretende establecer relaciones de } \\
\text { solidaridad y confianza con sus diferentes } \\
\text { públicos. } \\
\text { Por todo lo ante expuesto, se considera que el } \\
\text { modelo que sobresale en esta instalación es el } \\
\text { Modelo Simétrico Bidireccional con tendencia } \\
\text { a la escuela latinoamericana. }\end{array}$ & $\begin{array}{l}\text { El objetivo del trabajo del relacionista, es } \\
\text { la atención directa al es la atención directa } \\
\text { al cliente. Este, tiene un enfoque a corto } \\
\text { plazo y está explícito en planes y } \\
\text { programas. La naturaleza de la } \\
\text { comunicación es bidireccional y se } \\
\text { evidencia la retroalimentación mediante } \\
\text { encuestas, técnica que igualmente se } \\
\text { utilizan para realizar los estudios de } \\
\text { opinión pública. Existen relaciones con la } \\
\text { comunidad. } \\
\text { La disciplina no es considerada } \\
\text { mayoritariamente como una herramienta } \\
\text { de marketing y la organización, pretende } \\
\text { establecer relaciones de solidaridad y } \\
\text { confianza con sus diferentes públicos. } \\
\text { Por todo esto se considera que el modelo } \\
\text { que sobresale en esta instalación es el } \\
\text { Modelo Simétrico Bidireccional con } \\
\text { tendencia a la escuela Latinoamericana. }\end{array}$ \\
\hline
\end{tabular}

Cuadro No. 2. Resultados en Iberostar Varadero e Iberostar Parque Central . Fuente: Archivo de los autores

\subsection{ANÁLISIS DE LOS RESULTADOS}

Con respecto a la variable Generalidades de las Relaciones Públicas vemos que en los dos hoteles las Relaciones Públicas y sus objetivos se conocen, pero en el Iberostar 
Varadero se conocen más que en el Iberostar Parque Central, lo que puede deberse a decisiones específicas de la forma de administrar de los directores y líderes de cada hotel..

Con respecto a la variable se percibe que tienen en común la gestión en ambos hoteles la presencia de ocho de las 13 modalidades de Barreras Culturales en la Comunicación propuestas en el modelo elaborado por Chibás Ortiz (2005).

Se observó también una forma de expresión diferente de las mismas en cada hotel, tanto por su causa, carácter o naturaleza, así como por su forma de manifestación e intensidad. Destaca en el hotel Iberostar Parque Central, las Barreras Culturales en la Comunicación de Estilo autocrático de comunicación y Distancia Jerárquica, lo que puede deberse al descontento percibido en los trabajadores con los directivos actuales de y la manera de ellos hacer presente su gestión del liderazgo que trasciende al sentido de pertenencia de los trabajadores, tras un cambio de gerencia. De forma general las Barreras Culturales en la Comunicación fueron más intensas en el hotel Iberostar Parque Central de las que levantada en el Iberostar Varadero. Esto también puede deberse a que el último está más distante de ciudad e la Habana, donde radica la matriz de las redes Iberostar en Cuba y de la red Cuabancan y por eso tienen una relativa mayor libertad de gestión con respecto ala burocracia que el contacto con estas matrices requiere.

En el Hotel Iberostar Vardero las Barreras Culturales en la Comunicación que más se destacaron fueon las de Tendencia al inmediatismo y a sobrevalorar lo urbano con respecto a lo rural, lo cual se explica en el caso de la primera barrera ppor la urgencia en obtener resultados por parte de la gerencia y e el caso de la segunda por tener muchos funcionarios que son de origen rural que al trabajar en un hotel de primera pueden quedarse algo deslumbrados con el requinte y lujo que el mismo ofrece.

Las Barreras Culturales en la Comunicación que no se manifestaron en ambos hoteles fueron las de Tendencia al individualismo, Sexismo, Religiocentrismo, Relación destorcida con la ética y Tendencia a sobrevalorar la edad.

En la variable aspectos éticos se vio que ambos hoteles llevan estos elementos muy en serio, aún cuando los valores que más estimulen en un caso y otor sean algo diferentes. Destaca que en el hotel Iberostar Varadero se realizan cumpleaños colectivos y se insiste en la superación de los empleados. Se esfuerzan mucho por mantener un buen clima organizacional através de cumpleaños colectivos y otras actividades. Esto redunda 
en valores como solidaridad, respeto, confianza, fidelidad y cooperación están muy presentes.

Por su parte el hotel Iberostar Parque Central insiste más en la superación de los funcionarios como foco. Esto hace que los valores predominantes sean más los de conocimiento, respeto y confianza.

Las funciones del relacionista público en el hotel Iberostar Varadero se asocian más al papel de mediador de conflictos y gestor de la comunicación de la comunicación, en cuanto que en el hotel Iberostar Parque central a la de aseguramiento de una comunicación bidireccional y mantener una adecuada relación con las organizaciones políticas y de masas del país.

Por tratarse de un hotel a la orilla del mar el Iberostar Varadero exige del Perfil del relacionista público mucho carisma como diferencial. En el caso del Iberostar Parque Central, esta exigencia es menor.

Con respecto a la Segmentación de los públicos esta es muy bien definida por ambos hoteles.

En lo referente al ítem Investigación, se realizan estudios de opinión pública y auditorías de imagen, cada 3 meses, de forma patronizada por una compañía externa, aunque para esto el hotel Iberostar Vardero tiene una autonomía un poco mayor que le Iberostar Parque Central.

Con respecto a las acciones de relaciones públicas que se emprenden en ambos hoteles, podemos decir que en el hotel Iberostar Varadero se atribuyen su éxito a la forma de administrar, al clima organizacional positivo y a la presencia de recursos, mientras que en el Iberostar Parque Central se atribuye el éxito a la superación de los funcionarios, la, formación profesional de los trabajadores y dirigentes, las facilidades materiales, al estilo de dirección, a la correspondencia de prácticas culturales entre ambas gerencias y a las normas y leyes nacionales.

Ambos hoteles poseen una fuerte comunicación organizacional interna y externa, donde el relacionista público ejerce un grande papel. Pero la comunicación en el hotel Iberostar Varadero es menos piramidal o de arriba para abajo (de los directores para sus funcionarios, sino más en los dos sentidos), mientras que en el hotel Iberostar Parque Central, es más en estilo o orden descendente, es decir más de arriba para abajo o del directivos para sus funcionarios. 
El modelo de relaciones públicas adoptado en ambos hoteles es el Simétrico Bidireccional con tendencia a la escuela latinoamericana, según lo describe Kunsch (2006). Aún así se perciben diferencias, dado que en el hotel Iberostar Varadero se enfatiza mucho la relación con el huésped, pero también con el público interno de los funcionarios y la comunidad, mientras que en el Iberostar Parque Central el énfasis también envuelve la relación con las organizaciones políticas y de masa del país.

\section{CONCLUSIONES}

La inversión extranjera es un imponderable para el presente y futuro desarrollo de Cuba, con vistas a la actualización del Modelo Económico Cubano y su inserción en un mundo globalizado. Estudiar las regularidades y tendencias de su implementación constituye una tarea de obligado cumplimiento, en tanto se debe asegurar la defensa de los intereses nacionales, en medio de un contexto globalizador bastante convulso.

Las Relaciones Públicas constituyen una herramienta básica para la contribución al sentido de pertenencia, la identidad y la imagen de una institución. En este sentido, la comunicación intercultural es un proceso clave que opera en el sector del Turismo, particularmente en aquellas entidades cuya gerencia se ejecuta de manera multinacional, teniendo como brújula un adecuado intercambio de valores éticos y morales, así como el diagnóstico de las Barreras Culturales en la Comunicación .

El presente estudio y caracterización de la gestión de Relaciones Públicas de la Cadena Iberostar Hotels and Resorts ha permitido profundizar en un modelo cuyas particularidades pueden contribuir al perfeccionamiento de esta actividad en el resto del sector hotelero de explotación mixta.

Existen diferentes puntos de vista de la realidad, los prejuicios, opiniones y estereotipos, tanto entre trabajadores meramente nacionales, como entre nacionales y extranjeros. Estas Barreras Culturales en la Comunicación no se consideran en su totalidad de trascendencia para el éxito de la gestión organizacional; sin embargo, se expresan con bastante fuerza en orden de frecuencia: el sensualismo, la externalidad, el inmediatismo, el control de la incerteza y la distancia jerárquica.

Las claves del éxito se centran en el estilo de dirección, la política de estímulo, las facilidades materiales y la formación profesional. Se considera que los empresarios y 
trabajadores de procedencia española tienden a una rápida y adecuada adaptación al contexto cultural cubano.

Desde el punto de vista ético es importante decir que la Cadena Iberostar implementa las Relaciones Públicas en Cuba sin diferencias sustanciales con otros contextos sociopolíticos. Adapta su gestión en correspondencia con la modalidad que promueve cada entidad con un enfoque bidireccional de atención al cliente externo e interno. Trabaja bajo un código de ética que responde a la formación y difusión del compromiso, el respeto al trabajador, eficacia y la competencia organizacional. Se vincula y favorece la comunidad bajo principios de sustentabilidad y humanismo.

La Compañía percibe, de acurdo a los datos levantados en entrevistas, que la seguridad ciudadana y la hospitalidad son algunos de los valores fundamentales de la sociedad cubana. Los aspectos éticos de la gestión de la comunicación son ampliamente respetados, por esta red, razón por la cual enfrentan menos Barreras Culturales en la Comunicación en suelo cubano.

Otro aspecto que no feu lo suficientemente explorado por esta investigación, es el de la relación entre las Barreras Culturales en la Comunicación y la ética. Si se tienen más Barreras Culturales en la Comunicación es menos manifiesta la ética organizacional o al contrario, cuanto se tienen menos la relación entre las Barreras Culturales en la Comunicación, la ética organizacional menor es la manifestación de la ética empresarial? Preguntas estas que pueden ser abordadas en otras investigaciones.

Los resultados obtenidos en el presente trabajo, permitirán una evaluación oportuna de la forma de gestión empleada en las entidades de referencia para su posible perfeccionamiento y/o la generalización con cautela de sus buenas prácticas. El presente estudio es una investigación preliminar que debe en el futuro utilizar otras metodologías cuantitativas junto a las cualitativas que permitan metrificar mejor las diferencias y elementos analizados, así como extenderse por otros hoteles de esa misma red, además de posteriormente abarcar otras redes hoteleras presentes en el país para conseguir tener una "foto" más próxima de la realidad de las variables estudiadas.

\section{REFERENCIAS}

AGUILAR, M. G. La Comunicación en las organizaciones para la mejora de la productividad: El uso de los medios como fuente informativa en empresas e instituciones andaluzas. Universidad de Málaga. Málaga. 2007. 
ARREDONDO TRAPERO, Florina; GARZA GARCÍA, Jorge; VILLA CASTAÑA, Lida, Propuesta para el diseño de un código de ética empresarial basado en la ética kantiana, Cuadernos de Administración, Vol.30, No. 52, p. 9-19, jul-dec, 2014.

BÁEZ Deborah G. Trabajo de Diploma: "Las Relaciones Públicas en la gerencia multinacional turística de Cuba: un estudio de caso". Facultad de Turismo de la Universidad de la Habana. 2014.

DEBELJUH. Ética empresarial. En el núcleo de la estrategia corporativa. Editorial Cencage Learnin: Buenos Aires. 2009

GARCÍA, D. I. Relaciones publicas aplicadas a contextos turísticos. Aproximaciones al fenómeno desde la emergencia de un enfoque integrador. CIET: La Habana, 2012

HERNÁNDEZ, J. A. La necesidad de una conducta ética en el proceso administrativo. Una revisión de conceptos y perspectivas. Universidad del Valle de México. San Ángel: México, 2006.

KUNSCH, Margarida M. Krohling. Planejamento e gestão estratégica de relações públicas nas organizações contemporâneas. Análisis. No. 34, 2006.

MAGAlLÓN, S. Conceptos y Elementos de las Relaciones Públicas. Escola Superior de Relacions Públiques. Barcelona. 2006.

MURILLO, M., J Intervención del vicepresidente Marino Murillo Jorge en la Asamblea Nacional del Poder Popular correspondiente a la VIII Legislatura. Versión Taquigráfica del Consejo de Estado. La Habana. 2013.

MURUAGA, A.F; GUTIÉRREZ, A, G; MENDOZA, DIDIO Q; IZQUIERDO, VICTORIA, P. Cuba: Crisis, Ajuste y Situación Social 1990-1996. Editorial Ciencias Sociales, La Habana. 1998.

INFOTUR, Cuba bate el record de turistas, recibiendo 4 millones de turistas em 2016, 2017. Accesible en: http://www.infotur.cu Acceso: 28/04/2017.

ORTIZ. F. C. Barreras Culturales de la Comunicación y Relaciones Públicas, en redes hoteleras con sede en Sao Paulo. (Tesis de doctorado)Universidad de Sao Paulo. Sao Paulo. 2005.

Creatividad, Comunicación y Cultura. Gestión innovadora de proyectos educativo-culturales en la era digital. Editorial Pueblo y Educación. Cuba 2015.

ORTIZ, T. G. El Renacer de las Relaciones Públicas en Cuba. Editorial Academia. La Habana. 2001.

PATRUS-PENA, Roberto; CASTRO, Paula. Ética nos negócios, condições, desafios e riscos, São Paulo: Atlas, 2010.RIZO, M.; Romeu V., Cultura y Comunicación Intercultural. Aproximaciones conceptuales. 2006.

SAMPIERI, R. H. Metodología de la Investigación. Cuarta Edición. McGraw, Hill Interamericana. México D.F. 2006 
SANTOS, Fernando de Almeida. Ética Empresarial - Políticas de Responsabilidade Social Em 5 Dimensões, Atlas: São Paulo, 2014.

RUZ, R. C. Discurso Pronunciado por el Presidente de los Consejos de Estado y de Ministros, en las Conclusiones del XX Congreso de la Central de Trabajadores de Cuba. “Año 56 de la Revolución”. Versión Taquigráfica del Consejo de Estado. La Habana. 2014

TROCONES, L. M. Diagnóstico de la Gestión de las Relaciones Públicas en el Hotel Brisas Trinidad del Mar. Trabajo de Diploma. Facultad de Comunicación Social. Sancti Spíritus. Cuba. 2011.

TRELLES, I. Gestión de Comunicación Institucional: reflexiones en torno a un concepto y una praxis. Revista Espacio No. 10. Enero-Abril, 2005. 